\title{
Some Notes on Soft Topological Concepts and an Application Method
}

\author{
Mustafa Burç Kandemir*
}

\begin{abstract}
In this paper, using the concept of soft topology given in [9] i.e. with our new perspective of soft topology, we give some basic topological concepts such as open soft set, closed soft set, interior and closure of a soft set. We then give the concept of soft continuity of a given function between soft topological spaces, and from here we also define the concept of soft homeomorphism and argue the all obtained results. At the end of the article, we propose a decision-making method using soft topological concepts.
\end{abstract}

Keywords: Soft set; soft topological space; continuity; interior; closure.

AMS Subject Classification (2020): 03E99; 54C08.

*Corresponding author

\section{Introduction and Preliminaries}

The notion of soft set is defined by Molodtsov [13] in order to model uncertainties in science, engineering and human sciences and to solve the problems that arise within these concepts. He defined a soft set as a parameterization of some subsets the initial universal set. It's formal definition is as follows;

Definition 1.1. [13] Let $U$ be an initial universal set, $E$ be a parameters set, $A \subseteq E$. The pair $(F, A)$ is called a soft set over $U$ if $F: A \rightarrow \mathcal{P}(U)$ is a set-valued mapping where $\mathcal{P}(U)$ is a power set of $U$.

The foundations of soft set-theoretic operations are given in [11] and [3]. Now, let us give the operations we will use on this article.

Definition 1.2. [3,11] Let $(F, A)$ and $(G, B)$ be soft sets over $U$.

(1) $(G, B)$ is called a soft subset of $(F, A)$ if $B \subseteq A$ and $G(p) \subseteq F(p)$ for each $p \in B$, and denoted by $(G, B) \widetilde{C}(F, A)$. If $(F, A) \widetilde{C}(G, B)$ and $(G, B) \widetilde{C}(F, A)$, then it is called that $(F, A)$ is equal to $(G, B)$.

(2) The soft union of $(F, A)$ and $(G, B)$ is defined as the soft set $(H, C)$ over $U$, and denoted by $(F, A) \widetilde{\cup}(G, B)=$ $(H, C)$ such that $C=A \cup B$ and

$$
H(p)= \begin{cases}F(p) & , p \in A-B \\ G(p) & , p \in B-A \\ F(p) \cup G(p) & , p \in A \cap B\end{cases}
$$

for each $p \in C$.

(3) The soft intersection of $(F, A)$ and $(G, B)$ is defined as the soft set $(H, C)$ over $U$, and denoted by $(F, A) \widetilde{\cap}(G, B)=$ $(H, C)$ such that $C=A \cap B \neq \varnothing$ and $H(p)=F(p) \cap G(p)$ for each $p \in C$.

Received : 11-10-2019, Accepted : 16-02-2020 
(4) The complement of $(F, A)$ is defined and denoted as $(F, A)^{c}=\left(F^{c}, A\right)$ where $F^{c}: A \rightarrow \mathcal{P}(U)$ such that $F^{c}(p)=U-F(p)$ for each $p \in A$.

(5) It is called that $(F, A)$ is a relative whole soft set if $F(p)=U$ for each $p \in A$ and denoted by $\widetilde{\mathbb{U}}_{A}$. If $A=E,(F, E)$ is called absolute soft set and denoted by $\widetilde{\mathbb{U}}$. Similarly, $(F, A)$ is called a relative null soft set if $F(p)=\varnothing$ for each $p \in A$ and denoted by $\widetilde{\Phi}$.

We say that a soft set $(F, A)$ over the initial universe $U$ is a non-empty approximated soft set if $F(p) \neq \varnothing$ for each $p \in A$.

The notion of soft algebraic structure has been founded in [1] and [2]. In [2], soft groups have been defined and studied its properties. In [1], the concept of soft ring has been introduced and discussed its structural properties.

The concept of soft topology has been handled and studied by many scientists. In [16], the definition of soft topology was first given by Shabir and Naz on the initial universe with a fixed parameter set. They give the definition of their soft topology as follows;

Definition 1.3. [16] Let $\mathbb{T}$ be the collection of soft sets over $U$, then $\mathbb{T}$ is said to be a soft topology on $U$ if

(1) $\widetilde{\Phi}, \widetilde{\mathbb{U}}$ are in $\mathbb{T}$,

(2) the union of any number soft sets in $\mathbb{T}$ is in $\mathbb{T}$,

(3) the intersection of any two soft sets in $\mathbb{T}$ is in $\mathbb{T}$.

The triplet $(U, \mathbb{T}, E)$ is called a soft topological space over $U$.

They introduced the concepts of soft open set, soft closed set, soft closure, soft interior points, soft neighborhood of a point and soft separation axioms. Hussain and Ahmad have broadened the basic concepts given in [16] and examined the relevant properties in [7]. In [4], Aygünoğlu and Aygün investigated soft continuity of soft functions and introduced soft product spaces and compactness of soft topological spaces.

In [9], we discussed the notion of soft topology from a different standpoint than known. We believe that soft topology should carry the spirit of soft set. Therefore, we gave the concept of soft topology as a parametrization of the subspaces of a topological space, preserving Molodtsov's sense in [9].

Definition 1.4. [9] Let $(U, \mathcal{T})$ be a topological space, $E$ be a parameters set and $(F, A)$ be a soft set over $U$ where $A \subseteq E$. It is called that $(F, A)$ is a soft topology over $U$ if $\left(F(p), \mathcal{T}_{F(p)}\right)$ is a subspace of $(U, \mathcal{T})$ for each $p \in A$. $(F, A, \mathcal{T})$ is called a soft topological space over $U$.

We also gave some basic topological concepts such as Hausdorffness, compactness, connectedness for soft topological spaces and discussed their some properties in [9]. With the definitions in [9], we have not lost the soul of both topology and the soft set theory.

From Definition 1.4, obviously, we have following result.

Theorem 1.1. Let $(F, A)$ be a soft set over $U$ with parameter set $E$ where $\varnothing \neq A \subseteq E$. Then $\left(F, A, \mathcal{T}_{A}\right)$ is a soft topological space over a topological space $(U, \mathcal{T})$ iff $F(p) \neq \varnothing$ for each $p \in A$.

In this paper, some other basic topological concepts such as open soft set, closed soft set, interior and closure of a soft set, derived soft set, perfect and dense soft set. We also provide the terms pseudo-open soft set and pseudo-closed soft set which pertain to soft topology. Besides, we give the notion of continuity between two soft topological spaces and study its properties and defined the notion of soft homeomorphism. We especially use [14] and [17] for the basic topological concepts in the formation of this article.

At the end of the article, we propose a decision-making method using soft topological concepts.

\section{Some Basic Results}

In this section, we will give some basic topological concepts and the results that we have obtained.

Definition 2.1. Let $(U, \mathcal{T})$ be a topological space and $(F, A, \mathcal{T})$ be a soft topological space over $U$. Let $(G, B)$ be a soft subset of $(F, A)$. It is called that $(G, B)$ is an open soft set in $(F, A, \mathcal{T})$ if $G(p) \in \mathcal{T}_{F(p)}$ for each $p \in B$. 
Example 2.1. Let $U=\{a, b, c, d\}$ be a universal set, $\mathcal{T}=\{\varnothing, U,\{a, b\},\{b\}\}$ be a topology on $U$ and $E=\{1,2,3,4,5\}$ be a parameter set. Let $(F, A)=\{1=\{a, b\}, 2=\{c, d\}, 3=\{b, d\}\}$ be a soft topology such that $\mathcal{T}_{F(1)}=$ $\{\varnothing,\{a, b\},\{b\}\}, \mathcal{T}_{F(2)}=\{\varnothing,\{c, d\}\}$ and $\mathcal{T}_{F(3)}=\{\varnothing,\{b, d\},\{b\}\}$. Let $(G, B)=\{1=\varnothing, 3=\{b\}\}$ be a soft subset of $(F, A)$. Since $G(1)=\varnothing \in \mathcal{T}_{F(1)}$ for $1 \in B$ and $G(3) \in \mathcal{T}_{F(3)}$ for $3 \in B$, then $(G, B)$ is an open soft set in $(F, A, \mathcal{T})$.

Note that, the relative whole soft set in an overt soft topological space ${ }^{1}$ is an open soft set and the relative null soft set in any soft topological space is an open soft set. Since each soft set is its soft subset, it is an open soft set in the soft topological space defined on it.

Definition 2.2. Let $(U, \mathcal{T})$ be a topological space and $(F, A, \mathcal{T})$ be a soft topological space over $U$. Let $(G, B)$ be a soft subset of $(F, A)$. It is called that $(G, B)$ is a closed soft set in $(F, A, \mathcal{T})$ if $F(p)-G(p) \in \mathcal{T}_{F(p)}$ for each $p \in B$,i.e. the complement of $G(p)$ with respect to the subspace $F(p)$ is open for each $b \in B$. The complement of $G(p)$ with respect to $F(p)$ for each $p \in B$ is denoted by $(G(p))^{t}$.

Example 2.2. Consider the soft topological space given in Example 2.1, take the $(G, B)=\{1=\{a\}, 3=\{d\}\}$ soft subset of $(F, A)$. Since $(G(1))^{t}=(\{a\})^{t}=\{b\} \in \mathcal{T}_{F(1)}$ for $1 \in B$ and $(G(3))^{t}=\{d\}^{t}=\{b\} \in \mathcal{T}_{F(3)}$ for $3 \in B,(G, B)$ is a closed soft set in $(F, A, \mathcal{T})$.

Definition 2.3. Let $(U, \mathcal{T})$ be a topological space and $(F, A, \mathcal{T})$ be a soft topological space over $U$. Let $(G, B)$ be a soft subset of $(F, A)$. The soft set

$$
\widetilde{\mathfrak{i}}(G, B)=\{p=\mathfrak{i}(G(p)) \mid p \in B\}
$$

is called interior of $(G, B)$ in $(F, A, \mathcal{T})$ where $\mathfrak{i}(G(p))$ is an interior of $G(p)$ in the subspace $\left(F(p), \mathcal{T}_{F(p)}\right)$ for each $p \in B$.

Example 2.3. Let $(\mathbb{R}, \mathcal{U})$ be a usual topological space and $(F, A)=\{1=(0,1), 2=\mathbb{Z}, 3=\{1,2,3,4,5\}\}$ be a soft topology over $\mathbb{R}$ where $\mathcal{U}_{F(1)}=\mathcal{U}_{(0,1)}, \mathcal{U}_{F(2)}=\mathcal{P}(\mathbb{Z})$ and $\mathcal{U}_{F(3)}=\mathcal{P}(\{1,2,3,4,5\})$. Take $(G, B)=\left\{1=\left\{\frac{1}{2}\right\}, 2=\right.$ $\{3,4,5\}\}$ soft subset of $(F, A)$. Then $\widetilde{i}(G, B)=\{1=\varnothing, 2=\{3,4,5\}\}$.

Theorem 2.1. Let $(F, A, \mathcal{T})$ be a soft topological space over $(U, \mathcal{T})$ and $(G, B) \widetilde{C}(F, A)$. In this case the following properties are provided.

(1) $\widetilde{\mathfrak{i}}(G, B)$ is a soft union of all open soft set included by $(G, B)$.

(2) $\widetilde{\mathfrak{i}}(G, B) \widetilde{C}(G, B)$.

(3) $\widetilde{\mathfrak{i}}(G, B)$ is an open soft set.

(4) $(G, B)$ is an open soft set if and only if $\widetilde{\mathfrak{i}}(G, B)=(G, B)$.

Proof. (1) We know that interior of a set $X$ which is subset of any topological space $(U, \mathcal{T})$ is a union of all open sets included by $X$. So the proof is obvious from Definition 2.3.

(2) From Definition 2.3, we have $\mathfrak{i}(G(p)) \subseteq G(p)$ for each $p \in B$. Therefore, $\widetilde{\mathfrak{i}}(G, B) \widetilde{\subset}(G, B)$.

(3) From (1) and Definition 2.3, it is obvious.

(4) Suppose that $(G, B)$ is an open soft set in $(F, A, \mathcal{T})$. So $G(p) \in \mathcal{T}_{F(p)}$ for each $p \in B$ from Definition 2.1. In classical topology, we know that $G(p)=\mathfrak{i}(G(p))$ for each $p \in B$. Hence we obtain that $\widetilde{\mathfrak{i}}(G, B)=(G, B)$.

Conversely, suppose that $\widetilde{\mathfrak{i}}(G, B)=(G, B)$. From (3), $(G, B)$ is an open soft set.

Theorem 2.2. Let $(F, A, \mathcal{T})$ be a soft topological space over $(U, \mathcal{T})$ and $(G, B)$ and $(H, C)$ are two soft subsets of $(F, A)$. In this case the following properties are provided.

(1) $\widetilde{\mathfrak{i}}(\widetilde{\mathfrak{i}}(G, B))=\widetilde{\mathfrak{i}}(G, B)$.

(2) If $(G, B) \widetilde{\subset}(H, C)$, then $\widetilde{\mathfrak{i}}(G, B) \widetilde{\subset} \widetilde{\mathfrak{i}}(H, C)$.

(3) $\tilde{\mathfrak{i}}(G, B) \widetilde{\cup} \widetilde{\mathfrak{i}}(H, C) \widetilde{\subset} \widetilde{\mathfrak{i}}[(G, B) \widetilde{\cup}(H, C)]$.

\footnotetext{
${ }^{1}$ The soft topological space $\left(\widetilde{\mathbb{U}}_{A}, \mathcal{T}\right)$ is called the overt soft topological space over given any topological space $(U, \mathcal{T})$
} 
(4) $\widetilde{\mathfrak{i}}(G, B) \widetilde{\cap} \widetilde{\mathfrak{i}}(H, C)=\widetilde{\mathfrak{i}}[(G, B) \widetilde{\cap}(H, C)]$.

Proof. (1) From Theorem 2.1 (3), it is obvious.

(2) Since $(G, B) \widetilde{C}(H, C)$, we have $B \subseteq C$ and $G(p) \subseteq H(p)$ for each $p \in B$. So we have $\mathfrak{i}(G(p)) \subseteq \mathfrak{i}(H(p))$ for each $p \in B$. Then we obtain that $\widetilde{\mathfrak{i}}(G, B) \widetilde{\subset} \widetilde{\mathfrak{i}}(H, C)$.

(3) Let we say that $\widetilde{\mathfrak{i}}(G, B) \widetilde{\cup} \widetilde{\mathfrak{i}}(H, C)=(K, D)$. So, from the definition of soft union we have that $D=B \cup C$ and for each $p \in D$

$$
K(p)= \begin{cases}\mathfrak{i}(G(p)) & , p \in B-C \\ \mathfrak{i}(H(p)) & , p \in C-B \\ \mathfrak{i}(G(p)) \cup \mathfrak{i}(H(p)) & , p \in B \cap C\end{cases}
$$

Since $\mathfrak{i}(G(p)) \cup \mathfrak{i}(H(p)) \subseteq \mathfrak{i}(G(p) \cup H(p))$, from Equation (2.1) we obtain that

$$
\left\{\begin{array} { l l l } 
{ \mathfrak { i } ( G ( p ) ) } & { , p \in B - C } \\
{ \mathfrak { i } ( H ( p ) ) } & { , p \in C - B } \\
{ \mathfrak { i } ( G ( p ) ) \cup \mathfrak { i } ( H ( p ) ) } & { , p \in B \cap C }
\end{array} \subseteq \left\{\begin{array}{ll}
\mathfrak{i}(G(p)) & , p \in B-C \\
\mathfrak{i}(H(p)) & , p \in C-B \\
\mathfrak{i}(G(p) \cup H(p)) & , p \in B \cap C
\end{array}\right.\right.
$$

On the other hand, we say that $(G, B) \widetilde{\cup}(H, C)=(L, E)$, so $\widetilde{\mathfrak{i}}((G, B) \widetilde{\cup}(H, C))=\widetilde{\mathfrak{i}}(L, E)$. Here from and Definition 2.3 that $E=B \cup C$ and for each $p \in E$

$$
\mathfrak{i}(L(p))= \begin{cases}\mathfrak{i}(G(p)) & , p \in B-C \\ \mathfrak{i}(H(p)) & , p \in C-B \\ \mathfrak{i}(G(p) \cup H(p)) & , p \in B \cap C\end{cases}
$$

Hence we obtain that $D=E$ and $(K, D) \widetilde{\complement} \widetilde{i}(L, E)$ from right part of Equation (2.2) and Equation (2.3).

(4) It is straightforward from definition of soft intersection of two soft sets and Definition 2.3.

Definition 2.4. Let $(U, \mathcal{T})$ be a topological space and $(F, A, \mathcal{T})$ be a soft topological space over $U$. Let $(G, B)$ be a soft subset of $(F, A)$. The soft set

$$
\widetilde{\mathfrak{c}}(G, B)=\{p=\mathfrak{c}(G(p)) \mid p \in B\}
$$

is called closure of $(G, B)$ in $(F, A, \mathcal{T})$ where $\mathfrak{c}(G(p))$ is a closure of $G(p)$ in the subspace $\left(F(p), \mathcal{T}_{F(p)}\right)$ for each $p \in B$.

Example 2.4. Let $\mathbb{R}$ be the real number set as a universal set, $\mathcal{T}=\{\varnothing, \mathbb{R},[0,2]\}$ be a topology on $\mathbb{R}$ and $E=$ $\{1,2,3,4,5,6\}$ be the parameter set. Let

$$
(F, A)=\left\{1=(1,5], 2=\{\sqrt{2}\}, 4=\left(0, \frac{1}{2}\right)\right\}
$$

be a soft topology over $U$ where $\mathcal{T}_{F(1)}=\{\varnothing,(1,5],(1,2]\}, \mathcal{T}_{F(2)}=\{\varnothing,\{\sqrt{2}\}\}$ and $\mathcal{T}_{F(4)}=\left\{\varnothing,\left(0, \frac{1}{2}\right)\right\}$. Take $(G, B)=\left\{1=(2,4), 4=\left\{\frac{1}{3}, \frac{1}{4}\right\}\right\}$ subset of $(F, A)$, then we have the closure of $(G, B)$ as follows

$$
\widetilde{\mathfrak{c}}(G, B)=\left\{1=(2,5], 4=\left(0, \frac{1}{2}\right)\right\} .
$$

Theorem 2.3. Let $(F, A, \mathcal{T})$ be a soft topological space over $(U, \mathcal{T})$ and $(G, B) \widetilde{C}(F, A)$. In this case the following properties are provided.

(1) $\mathfrak{\mathfrak { c }}(G, B)$ is a soft intersection of all closed soft set containing $(G, B)$.

(2) $(G, B) \widetilde{\subset} \widetilde{c}(G, B)$.

(3) $\widetilde{\mathfrak{c}}(G, B)$ is a closed soft set.

(4) $(G, B)$ is a closed soft set if and only if $(G, B)=\widetilde{\mathfrak{c}}(G, B)$.

Proof. The proof is straightforward.

Theorem 2.4. Let $(F, A, \mathcal{T})$ be a soft topological space over $(U, \mathcal{T})$ and $(G, B)$ and $(H, C)$ are two soft subsets of $(F, A)$. In this case the following properties are provided. 
(1) $\tilde{\mathfrak{c}}(\mathfrak{c}(G, B))=\widetilde{\mathfrak{c}}(G, B)$.

(2) If $(G, B) \widetilde{\complement}(H, C)$, then $\widetilde{\mathfrak{c}}(G, B) \widetilde{\subset} \widetilde{\mathfrak{c}}(H, C)$.

(3) $\widetilde{\mathfrak{c}}((G, B) \widetilde{\cup}(H, C))=\widetilde{\mathfrak{c}}(G, B) \widetilde{\cup} \widetilde{\mathfrak{c}}(H, C)$.

(4) $\widetilde{\mathfrak{c}}((G, B) \widetilde{\cap}(H, C)) \widetilde{\subset} \widetilde{\mathfrak{c}}(G, B) \widetilde{\cap} \widetilde{\mathfrak{c}}(H, C)$.

Proof. (1) Since $\mathfrak{c}(\mathfrak{c}(G(p)))=\mathfrak{c}(G(p))$ for each $p \in B$, we have $\widetilde{\mathfrak{c}}(\widetilde{\mathfrak{c}}(G, B))=\widetilde{\mathfrak{c}}(G, B)$ from Definition 2.4.

Proofs of (2), (3) and (4) are straightforward similar to (1).

Definition 2.5. Let $(U, \mathcal{T})$ be a topological space and $(F, A, \mathcal{T})$ be a soft topological space over $U$. Let $(G, B)$ be a soft subset of $(F, A)$. The soft set

$$
(G, B)^{\prime}=\left\{p=(G(p))^{\prime} \mid p \in B\right\}
$$

is called derived soft set of $(G, B)$ where $(G(p))^{\prime}$ is the derived set of $G(p)$ in the subspace $\left(F(p), \mathcal{T}_{F(p)}\right)$ for each $p \in B$.

Example 2.5. Let $U=\{a, b, c\}$ be a universal set, $\mathcal{T}=\{\varnothing, U,\{a\}\}$ be a topology on $U$ and $E=\{1,2,3,4,5,6\}$ be a parameter set. Let $(F, A)=\{2=\{a, b\}, 4=U\}$ be a soft topology where $\mathcal{T}_{F(2)}=\{\varnothing,\{a, b\},\{a\}\}$ and $\mathcal{T}_{F(4)}=\mathcal{T}$. Consider the soft set $(G, B)=\{2=\{a, b\}\}$ which is soft subset of $(F, A)$. Then we obtain that $(G, B)^{\prime}=\{2=\{b\}\}$.

Theorem 2.5. Let $(F, A, \mathcal{T})$ be a soft topological space over $(U, \mathcal{T})$ and $(G, B)$ and $(H, C)$ are two soft subsets of $(F, A)$. In this case the following properties are provided.

(1) If $(G, B) \widetilde{C}(H, C)$ then $(G, B)^{\prime} \widetilde{\subset}(H, C)^{\prime}$.

(2) $\widetilde{\mathfrak{c}}(G, B)=(G, B) \widetilde{\cup}(G, B)^{\prime}$.

(3) $(G, B)$ is closed soft set if and only if $(G, B)^{\prime} \widetilde{\subset}(G, B)$.

(4) $((G, B) \widetilde{\cup}(H, C))^{\prime}=(G, B)^{\prime} \widetilde{\cup}(H, C)^{\prime}$.

Proof. The proof is straightforward.

Definition 2.6. Let $(F, A, \mathcal{T})$ be a soft topological space over $(U, \mathcal{T})$ and $(G, B)$ is a soft subset of $(F, A)$. We call that $(G, B)$ is a perfect soft set if $(G, B)^{\prime}=(G, B)$.

Definition 2.7. Let $(F, A, \mathcal{T})$ be a soft topological space over $(U, \mathcal{T})$ and $(G, B)$ is a soft subset of $(F, A)$. We call that $(G, B)$ is a dense soft set if $\mathfrak{i}(\mathfrak{c}(G(p))) \neq \varnothing$ in $\left(F(p), \mathcal{T}_{F(p)}\right)$ for each $p \in B$.

Note that, $\widetilde{\mathfrak{i}}(\widetilde{\mathfrak{c}}(G, B)) \neq \widetilde{\Phi}$ is not enough for $(G, B)$ to be dense.

Definition 2.8. Let $(F, A, \mathcal{T})$ be a soft topological space over $(U, \mathcal{T})$ and $(G, B)$ is a soft subset of $(F, A)$. We call that $(G, B)$ is a everywhere dense soft set if $\widetilde{\mathfrak{c}}(G, B)=\left(\left.F\right|_{B}, B\right)$.

Definition 2.9. Let $(F, A, \mathcal{T})$ be a soft topological space over $(U, \mathcal{T})$ and $(G, B)$ is a soft subset of $(F, A)$. We call that $(G, B)$ is a nowhere dense soft set if $\widetilde{\mathfrak{i}}(\widetilde{\mathfrak{c}}(G, B))=\widetilde{\Phi}$.

Example 2.6. Consider the given in Example 2.4. we compute the interior of $\widetilde{\mathfrak{c}}(G, B)$ as

$$
\widetilde{\mathfrak{i}}(\widetilde{\mathfrak{c}}(G, B))=\left\{1=\varnothing, 4=\left(0, \frac{1}{2}\right)\right\} \neq \widetilde{\Phi} .
$$

But it is not dense. We take the soft set $(H, C)=\left\{1=(1,2), 4=\left\{\frac{1}{3}, \frac{1}{4}\right\}\right\}$ which is soft subset of $(F, A)$. Then we have

$$
\widetilde{\mathfrak{i}} \mathfrak{c}(G, B))=\left\{1=(1,5], 4=\left(0, \frac{1}{2}\right)\right\}
$$

and we obtain that $\widetilde{\mathfrak{i}}(\widetilde{\mathfrak{c}}(G(p))) \neq \varnothing$ in $\left(F(p), \mathcal{T}_{F(p)}\right)$ for each $p \in B$, so it is dense soft set in $(F, A, \mathcal{T})$. 
Example 2.7. Let $\mathcal{T}=\{\varnothing, \mathbb{R},[0,2]\}$ be a topology on the real number $\mathbb{R}$ and $E=\{1,2,3,4,5\}$. Let $(F, A)=$ $\{1=(1,5], 2=\{\sqrt{2}\}, 4=(1,3)\}$ be a soft topology where $\mathcal{T}_{F(1)}=\{\varnothing,(1,5],(1,2]\}, \mathcal{T}_{F(2)}=\{\varnothing,\{\sqrt{2}\}\}$ and $\mathcal{T}_{F(4)}=\{\varnothing,(1,3),(1,2]\}$. Consider the soft set $(G, B)=\left\{1=(2,4), 4=\left\{\frac{5}{2}\right\}\right\}$ soft subset of $(F, A)$. So we have

$$
\widetilde{\mathfrak{c}}(G, B)=\{1=(2,5], 4=(2,3)\} .
$$

Hence we obtain that

$$
\widetilde{\mathfrak{i}}(\widetilde{\mathfrak{c}}(G, B))=\{1=\varnothing, 4=\varnothing\}=\widetilde{\Phi}_{B} .
$$

Thus $(G, B)$ is a nowhere dense soft set in $(F, A, \mathcal{T})$.

Definition 2.10. Let $(F, A, \mathcal{T})$ be a soft topological space over $(U, \mathcal{T})$, and $(G, B)$ be a soft subset of $(F, A)$. $(G, B)$ is called pseudo-open soft set if there exists $p \in B$ such that $G(p) \in \mathcal{T}_{F(p)}$.

Similarly, we call that $(G, B)$ is a pseudo-closed soft set if there exists $p \in B$ such that $G(p)$ is closed in $\left(F(p), \mathcal{T}_{F(p)}\right)$.

Note that each open soft set (closed soft set) is a pseudo-open soft set (pseudo-closed soft set).

Example 2.8. Let $U=\{a, b, c, d, e\}$ be a universal set, $\mathcal{T}=\{\varnothing, U,\{a\},\{a, b, d\}\}$ be a topology on $U$ and $E=$ $\{1,2,3,4,5,6\}$ be a parameters set. Let $(F, A)=\{1=\{a, d, e\}, 3=\{b\}, 4=\{a, c\}\}$ be a soft topology such that $\mathcal{T}_{F(1)}=\{\varnothing,\{a, d, e\},\{a\},\{a, d\}\}, \mathcal{T}_{F(3)}=\{\varnothing,\{b\}\}$ and $\mathcal{T}_{F(4)}=\{\varnothing,\{a, c\},\{a\}\}$. Let $(G, B)=\{1=\{a, d\}, 4=\{c\}\}$ be soft subset of $(F, A)$. Hence $(G, B)$ is a pseudo-open soft set over $U$.

Theorem 2.6. If $(G, B)$ and $(H, C)$ are pseudo-open (pseudo-closed) soft sets with respect to the soft topological sapces $(F, A, \mathcal{T})$, then $(G, B) \widetilde{\cup}(H, C)$ is a pseudo-open (pseudo-closed) soft set.

Proof. From Definition 1.2, we have $(G, B) \widetilde{\cup}(H, C)=(K, D)$ such that $D=B \cup C$ and $K(p)=G(p)$ if $p \in B-C$, $K(p)=H(p)$ if $p \in C-B$ and $G(p) \cup H(p)$ if $p \in B \cap C$. The proof for the first two cases is obvious. The last case, since both $G(p)$ and $H(p)$ open for $p \in B \cap C$, their union $G(p) \cup H(p)$ is open. Hence $(K, D)$ is a pseudo-open soft set over $U$.

The same is true for pseudo-closed soft set.

Note that, the soft intersection of pseudo-open (pseudo-closed) soft sets may not be pseudo-open (pseudo-closed). We can give the following example for this.

Example 2.9. Let' $\mathrm{s}$ use the arguments in Example 2.8. We know that $(G, B)$ is a pseudo-open soft set. Let $(H, C)=\{3=\{b\}, 4=\{c\}\}$ be a soft subset of $(F, A)$ which is pseudo-open. Then their soft intersection is $(K, D)=\{4=\{c\}\}$ and it is not pseudo-open soft set over $U$ with respect to $(F, A, \mathcal{T})$.

\section{Continuity in Soft Topological Spaces}

The concept of continuity is one of the most important topics of topology. In this section, we give the concept of continuity of a function between soft topological space. Let $(U, \mathcal{T})$ and $\left(V, \mathcal{T}^{\prime}\right)$ be two topological space and $\varphi: U \rightarrow V$ be a function. Let $(F, A, \mathcal{T})$ and $\left(G, B, \mathcal{T}^{\prime}\right)$ be soft topological spaces over $U$ and $V$, respectively. We denote that $\varphi_{p, p^{\prime}}:\left(F(p), \mathcal{T}_{F(p)}\right) \rightarrow\left(G\left(p^{\prime}\right), \mathcal{T}_{G\left(p^{\prime}\right)}^{\prime}\right)$ for each $p \in A$ and $p^{\prime} \in B$. Then, we define the concept of continuity between soft topological spaces as follows.

Definition 3.1. (1) $\varphi$ is called soft pseudo-continuous function from $(F, A, \mathcal{T})$ to $\left(G, B, \mathcal{T}^{\prime}\right)$ if there exist $p \in A$ and $p^{\prime} \in B$ such that $\varphi_{p, p^{\prime}}$ is a continuous from $\left(F(p), \mathcal{T}_{F(p)}\right)$ to $\left(G\left(p^{\prime}\right), \mathcal{T}_{G\left(p^{\prime}\right)}^{\prime}\right)$.

(2) $\varphi$ is called soft continuous function from $(F, A, \mathcal{T})$ to $\left(G, B, \mathcal{T}^{\prime}\right)$ if for all $p \in A$ and $p^{\prime} \in B, \varphi_{p, p^{\prime}}$ is continuous from $\left(F(p), \mathcal{T}_{F(p)}\right)$ to $\left(G\left(p^{\prime}\right), \mathcal{T}_{G\left(p^{\prime}\right)}^{\prime}\right)$.

Example 3.1. Let $U=\{a, b, c\}$ and $V=\{d, e, f, g, h\}$ be universal sets and $\mathcal{T}=\{\varnothing, U,\{a, b\},\{a\}\}$ and $\mathcal{T}^{\prime}=$ $\{\varnothing, V,\{d, e\},\{f, g\},\{d, e, f, g\}\}$ be topologies on $U$ and $V$, respectively. Let $(F, A)=\{1=\{a, c\}, 2=\{a\}\}$ be a soft topology over $U$ where $\mathcal{T}_{F(1)}=\{\varnothing,\{a, c\},\{a\}\}$ and $\mathcal{T}_{F(2)}=\{\varnothing,\{a\}\}$. Let $(G, B)=\{\triangle=\{d, e\}, \square=\{g\}\}$ be a soft topology over $V$ where $\mathcal{T}_{G(\triangle)}^{\prime}=\{\varnothing,\{d, e\}\}$ and $\mathcal{T}_{G(\square)}^{\prime}=\{\varnothing,\{g\}\}$.

Consider the function $\varphi: U \rightarrow V$ such that $\varphi=\{(a, d),(b, e),(c, g)\}$. Since

$\varnothing \in \mathcal{T}_{G(\triangle)}^{\prime} \Rightarrow \varphi_{1, \triangle}^{-1}[\varnothing]=\varnothing \in \mathcal{T}_{F(1)},\{d, e\} \in \mathcal{T}_{G(\triangle)}^{\prime} \Rightarrow \varphi_{1, \triangle}^{-1}[\{d, e\}]=\{a, b\} \notin \mathcal{T}_{F(1)}$,

$\varnothing \in \mathcal{T}_{G(\square)}^{\prime} \Rightarrow \varphi_{1, \square}^{-1}[\varnothing]=\varnothing \in \mathcal{T}_{F(1)},\{g\} \in \mathcal{T}_{G(\square)}^{\prime} \Rightarrow \varphi_{1, \square}^{-1}[\{g\}]=\{c\} \notin \mathcal{T}_{F(1)}$, 
$\varnothing \in \mathcal{T}_{G(\triangle)}^{\prime} \Rightarrow \varphi_{2, \triangle}^{-1}[\varnothing]=\varnothing \in \mathcal{T}_{F(2)},\{d, e\} \in \mathcal{T}_{G(\triangle)}^{\prime} \Rightarrow \varphi_{2, \Delta}^{-1}[\{d, e\}]=\{a, b\} \notin \mathcal{T}_{F(2)}$,

$\varnothing \in \mathcal{T}_{G(\square)}^{\prime} \Rightarrow \varphi_{2, \square}^{-1}[\varnothing]=\varnothing \in \mathcal{T}_{F(2)},\{d, e\} \in \mathcal{T}_{G(\square)}^{\prime} \Rightarrow \varphi_{2, \square}^{-1}[\{g\}]=\{c\} \notin \mathcal{T}_{F(2)}, \varphi$ is neither soft pseudocontinuous nor soft continuous.

Consider the function $\phi: U \rightarrow V$ such that $\phi=\{(a, g),(b, f),(c, f)\}$. Since

$\varnothing \in \mathcal{T}_{G(\triangle)}^{\prime} \Rightarrow \phi_{1, \triangle}^{-1}[\varnothing]=\varnothing \in \mathcal{T}_{F(1)},\{d, e\} \in \mathcal{T}_{G(\triangle)}^{\prime} \Rightarrow \phi_{1, \triangle}^{-1}[\{d, e\}]=\varnothing \in \mathcal{T}_{F(1)}$,

$\varnothing \in \mathcal{T}_{G(\square)}^{\prime} \Rightarrow \phi_{1, \square}^{-1}[\varnothing]=\varnothing \in \mathcal{T}_{F(1)},\{g\} \in \mathcal{T}_{G(\square)}^{\prime} \Rightarrow \phi_{1, \square}^{-1}[\{g\}]=\{a\} \in \mathcal{T}_{F(1)}$,

$\varnothing \in \mathcal{T}_{G(\triangle)}^{\prime} \Rightarrow \phi_{2, \triangle}^{-1}[\varnothing]=\varnothing \in \mathcal{T}_{F(2)},\{d, e\} \in \mathcal{T}_{G(\triangle)}^{\prime} \Rightarrow \phi_{2, \Delta}^{-1}[\{d, e\}]=\varnothing \in \mathcal{T}_{F(2)}$,

$\varnothing \in \mathcal{T}_{G(\square)}^{\prime} \Rightarrow \phi_{2, \square}^{-1}[\varnothing]=\varnothing \in \mathcal{T}_{F(2)},\{g\} \in \mathcal{T}_{G(\square)}^{\prime} \Rightarrow \phi_{2, \square}^{-1}[\{g\}]=\{a\} \in \mathcal{T}_{F(2)}, \phi$ is a soft continuous function from $(F, A, \mathcal{T})$ to $\left(G, B, \mathcal{T}^{\prime}\right)$.

Now, consider the function $\psi: U \rightarrow V$ such that $\psi=\{(a, e),(b, g),(c, e)\}$. So,

$\varnothing \in \mathcal{T}_{G(\triangle)}^{\prime} \Rightarrow \psi_{1, \triangle}^{-1}[\varnothing]=\varnothing \in \mathcal{T}_{F(1)},\{d, e\} \in \mathcal{T}_{G(\triangle)}^{\prime} \Rightarrow \psi_{1, \triangle}^{-1}[\{d, e\}]=\{a, c\} \in \mathcal{T}_{F(1)}$,

$\varnothing \in \mathcal{T}_{G(\square)}^{\prime} \Rightarrow \psi_{1, \square}^{-1}[\varnothing]=\varnothing \in \mathcal{T}_{F(1)},\{g\} \in \mathcal{T}_{G(\square)}^{\prime} \Rightarrow \psi_{1, \square}^{-1}[\{g\}]=\{b\} \notin \mathcal{T}_{F(1)}$,

$\varnothing \in \mathcal{T}_{G(\triangle)}^{\prime} \Rightarrow \psi_{2, \triangle}^{-1}[\varnothing]=\varnothing \in \mathcal{T}_{F(2)},\{d, e\} \in \mathcal{T}_{G(\triangle)}^{\prime} \Rightarrow \psi_{2, \Delta}^{-1}[\{d, e\}]=\{a, c\} \notin \mathcal{T}_{F(2)}$,

$\varnothing \in \mathcal{T}_{G(\square)}^{\prime} \Rightarrow \psi_{2, \square}^{-1}[\varnothing]=\varnothing \in \mathcal{T}_{F(2)},\{g\} \in \mathcal{T}_{G(\square)}^{\prime} \Rightarrow \psi_{2, \square}^{-1}[\{g\}]=\{b\} \notin \mathcal{T}_{F(2)}$. Since only $\psi_{1, \triangle}$ is continuous for $1 \in A, \triangle \in B, \psi$ is a soft pseudo-continuous function from $(F, A, \mathcal{T})$ to $\left(G, B, \mathcal{T}^{\prime}\right)$.

Note that, $\varphi:(U, \mathcal{T}) \rightarrow\left(V, \mathcal{T}^{\prime}\right)$ need not to be continuous as given in Example 3.1.

Obviously, all soft continuous function is a soft pseudo-continuous function.

Definition 3.2. Let $(F, A, \mathcal{T})$ and $\left(G, B, \mathcal{T}^{\prime}\right)$ be soft topological spaces over the topological space $(U, \mathcal{T})$ and $\left(V, \mathcal{T}^{\prime}\right)$, respectively. For the function $\varphi:(F, A, \mathcal{T}) \rightarrow\left(G, B, \mathcal{T}^{\prime}\right)$, we define the preimage of $(H, C)$ under $\varphi$ is a soft subset $(L, D)$ of $(F, A)$ where there exist $D \subseteq A$ and for all $p^{\prime} \in C$ there exist $p \in D$ such that $\varphi^{-1}\left[H\left(p^{\prime}\right)\right]=L(p)$, and denoted by $\varphi^{-1}[(H, C)]=(L, D)$.

Lemma 3.1. Let $(F, A, \mathcal{T})$ and $\left(G, B, \mathcal{T}^{\prime}\right)$ be soft topological spaces. Let $(H, C)$ and $(K, D)$ be soft subsets of $(G, B)$, if $(H, C) \widetilde{\complement}(K, D)$ then $\varphi^{-1}[(H, C)] \widetilde{\subset} \varphi^{-1}[(K, D)]$.

Proof. It is straightforward.

Theorem 3.1. $\varphi$ is soft continuous if and only if $\varphi^{-1}[(H, C)]$ is an open soft set in $(F, A, \mathcal{T})$ for each open soft set $(H, C)$ in $\left(G, B, \mathcal{T}^{\prime}\right)$.

Proof. Suppose that $\varphi$ is a soft continuous function from $(F, A, \mathcal{T})$ to $\left(G, B, \mathcal{T}^{\prime}\right)$. From Definition 3.1, we have that for all $p \in A$ there exist $p^{\prime} \in B$ such that $\varphi_{p, p^{\prime}}$ is a continuous function from $\left(F(p), \mathcal{T}_{F(p)}\right)$ to $\left(G\left(p^{\prime}\right), \mathcal{T}_{G\left(p^{\prime}\right)}^{\prime}\right)$. Take an arbitrary open soft subset $(H, C)$ of $(G, B)$. So from Definition 3.2, there exist the soft subset $(L, D)$ of $(F, A)$ such that $\varphi^{-1}[(H, C)]=(L, D)$. So we have that for all $p^{\prime} \in C$ there exist $p \in D \subseteq A$ such that $\varphi^{-1}\left[\left(H\left(p^{\prime}\right)\right)\right]=L(p)$. Since $\varphi$ is soft continuous and $(H, C)$ is an open soft set, then $\varphi^{-1}\left[H\left(p^{\prime}\right)\right]=L(p) \in \mathcal{T}_{F(p)}$ for each $p \in D$. Thus $(L, D)$ is an open soft set in $(F, A, \mathcal{T})$.

Conversely, suppose that $\varphi^{-1}[(H, C)]$ is an open soft set in $(F, A, \mathcal{T})$ for each open soft set $(H, C)$ in $\left(G, B, \mathcal{T}^{\prime}\right)$. From Definition 3.2, we have an open soft subset $(L, D)$ of $(F, A)$ such that $\varphi^{-1}[(H, C)]=(L, D)$, i.e. for each $p \in D$, $\varphi^{-1}\left(H\left(p^{\prime}\right)\right)=L(p) \in \mathcal{T}_{F(p)}$. Since $(H, C)$ is an arbitrary open soft subset of $(G, B)$, for each $C \subset B$, there is $D \subseteq A$. So for each $p \in A$ there is a $p^{\prime} \in B$ such that $\varphi_{p, p^{\prime}}$ is a continuous function from $\left(F(p), \mathcal{T}_{F(p)}\right)$ to $\left(G\left(p^{\prime}\right), \mathcal{T}_{G\left(p^{\prime}\right)}\right)$. Hence $\varphi$ is a soft continuous function from $(F, A, \mathcal{T})$ to $\left(G, B, \mathcal{T}^{\prime}\right)$.

Corollary 3.1. $\varphi$ is soft continuous if and only if $\varphi^{-1}[(H, C)]$ is a closed soft set in $(F, A, \mathcal{T})$ for each closed soft set $(H, C)$ in $\left(G, B, \mathcal{T}^{\prime}\right)$.

Theorem 3.2. $\varphi:(F, A, \mathcal{T}) \rightarrow(G, B, \mathcal{T})$ is a soft continuous function if and only if $\varphi^{-1}[\widetilde{\mathfrak{i}}(H, C)] \widetilde{\subset} \widetilde{\mathfrak{i}}\left(\varphi^{-1}[(H, C)]\right)$ for each $(H, C) \widetilde{C}(G, B)$.

Proof. Suppose that $\varphi$ is a soft continuous function. Take arbitrary $(H, C) \widetilde{C}(G, B)$. From Theorem $2.1(3), \widetilde{\mathfrak{i}}(H, C)$ is an open soft set in $\left(G, B, \mathcal{T}^{\prime}\right)$. Since $\varphi$ is soft continuous, we have $\varphi^{-1}[\widetilde{\mathfrak{i}}(H, C)]$ is an open soft set in $(F, A, \mathcal{T})$. From Theorem 2.1 (4), we have

$$
\widetilde{\mathfrak{i}}\left(\varphi^{-1}[\widetilde{\mathfrak{i}}(H, C)]\right)=\varphi^{-1}[\widetilde{\mathfrak{i}}(H, C)] .
$$


Since $\widetilde{\mathfrak{i}}(H, C) \widetilde{C}(H, C)$ from Theorem 2.1 (2), we have $\varphi^{-1}[\widetilde{\mathfrak{i}}(H, C)] \widetilde{\subset} \varphi^{-1}[(H, C)]$ from Lemma 3.1. So we have $\widetilde{\mathfrak{i}}\left(\varphi^{-1}[\widetilde{\mathfrak{i}}(H, C)]\right) \widetilde{\subset} \widetilde{\mathfrak{i}}\left(\varphi^{-1}[(H, C)]\right)$ from Theorem $2.2(2)$. Thus we have

$$
\varphi^{-1}[\widetilde{\mathfrak{i}}(H, C)] \widetilde{\subset} \widetilde{\mathfrak{i}}\left(\varphi^{-1}[(H, C)]\right)
$$

from Equation (3.1).

On the other hand, suppose that the condition $\varphi^{-1}[\widetilde{\mathfrak{i}}(H, C)] \widetilde{\subset} \widetilde{\mathfrak{i}}\left(\varphi^{-1}[(H, C)]\right)$ is satisfied for each $(H, C) \widetilde{\subset}(G, B)$. Let $(H, C)$ be an open soft set in $\left(G, B, \mathcal{T}^{\prime}\right)$. Then, we have that

$$
\varphi^{-1}[\widetilde{\mathfrak{i}}(H, C)]=\varphi^{-1}[(H, C)] \widetilde{\subset} \widetilde{\mathfrak{i}}\left(\varphi^{-1}[(H, C)]\right) \quad \widetilde{\subset} \varphi^{-1}[(H, C)] .
$$

Therefore $\varphi^{-1}[(H, C)]=\widetilde{\mathfrak{i}}\left(\varphi^{-1}[(H, C)]\right)$, and so $\varphi^{-1}[(H, C)]$ is an open soft set from Theorem $2.1(4)$. Thus $\varphi$ is a continuous function from Theorem 3.1.

Theorem 3.3. $\varphi:(F, A, \mathcal{T}) \rightarrow(G, B, \mathcal{T})$ is a soft continuous function if and only if $\widetilde{\mathfrak{c}}\left(\varphi^{-1}[(H, C)]\right) \widetilde{\subset} \varphi^{-1}[\widetilde{\mathfrak{c}}(H, C)]$ for each $(H, C) \widetilde{\subset}(G, B)$.

Proof. Suppose that $\varphi$ is continuous. Since $\widetilde{\mathfrak{c}}(H, C)$ is closed from Theorem $2.3(3)$ and $\varphi$ is continuous, $\varphi^{-1}[\widetilde{\mathfrak{c}}(H, C)]$ is closed soft set in $(F, A, \mathcal{T})$ from Corollary 3.1. Since $(H, C) \widetilde{\subset} \widetilde{\mathfrak{c}}(H, C)$, we have $\left.\varphi^{-1}[(H, C)] \widetilde{\subset} \varphi^{-1} \widetilde{\mathfrak{c}}(H, C)\right]$ from Lemma 3.1. Consequently, we obtain that

$$
\widetilde{\mathfrak{c}}\left(\varphi^{-1}[(H, C)]\right) \quad \widetilde{\subset} \varphi^{-1}[\widetilde{\mathfrak{c}}(H, C)]
$$

from Theorem 2.3 (1).

Conversely, let $(H, C)$ be a closed soft set in $\left(G, B, \mathcal{T}^{\prime}\right)$. So, $\widetilde{\mathfrak{c}}(H, C)=(H, C)$ from Theorem 2.3 (4). Then we have

$$
\left.\varphi^{-1}[(H, C)] \widetilde{\subset} \widetilde{\mathfrak{c}}\left(\varphi^{-1}[(H, C)]\right) \widetilde{\subset} \varphi^{-1} \widetilde{\mathfrak{c}}(H, C)\right]=\varphi^{-1}[(H, C)] .
$$

Thus $\varphi^{-1}[(H, C)]$ is a closed soft set in $(F, A, \mathcal{T})$. Hence we obtain that $\varphi$ is continuous from Corollary 3.1.

Theorem 3.4. Let $\varphi:(U, \mathcal{T}) \rightarrow\left(V, \mathcal{T}^{\prime}\right)$ and $\psi:\left(V, \mathcal{T}^{\prime}\right) \rightarrow\left(W, \mathcal{T}^{\prime \prime}\right)$ be functions. Let $(F, A, \mathcal{T}),\left(G, B, \mathcal{T}^{\prime}\right)$ and $\left(H, C, \mathcal{T}^{\prime \prime}\right)$ be soft topological spaces over $U, V$ and $W$, respectively. If $\varphi:(F, A, \mathcal{T}) \rightarrow\left(G, B, \mathcal{T}^{\prime}\right)$ and $\psi:\left(G, B, \mathcal{T}^{\prime}\right) \rightarrow\left(H, C, \mathcal{T}^{\prime \prime}\right)$ are soft continuous functions, then their composition $\psi \circ \phi$ is soft continuous from $(F, A, \mathcal{T})$ to $\left(H, C, \mathcal{T}^{\prime \prime}\right)$.

Proof. Take an arbitrary open soft set $(K, D)$ from $\left(H, C, \mathcal{T}^{\prime \prime}\right)$. Since $\psi$ is soft continuous, then $\psi^{-1}[(H, C)]$ is an open soft set in $\left(G, B, \mathcal{T}^{\prime}\right)$ from Theorem 3.1. Since $\varphi$ is soft continuous, the same operation is applied to $\psi^{-1}[(H, C)]$, we have that

$$
\varphi^{-1}\left[\psi^{-1}[(H, C)]\right]=(\psi \circ \varphi)^{-1}[(H, C)]
$$

is an open soft set in $(F, A, \mathcal{T})$. Thus $\psi \circ \varphi$ is a soft continuous function from Theorem 3.1.

Note that, id $:(U, \mathcal{T}) \rightarrow(U, \mathcal{T})$ be an identity function. Let $(F, A, \mathcal{T})$ be a soft topological space over $U$. The identity soft function id $:(F, A, \mathcal{T}) \rightarrow(F, A, \mathcal{T})$ is not a soft continuous function. For example,

Example 3.2. Let $U=\{a, b, c, d\}$ be a set , $\mathcal{T}=\{\varnothing, U,\{a\},\{b\},\{a, b\}\}$ be a topology on $U, E=\{1,2,3,4,5\}$ be the parameter set and $(F, A)=\{1=\{c, d\}, 2=\{a, c\},\{b, d\}\}$ be a soft topology where $\mathcal{T}_{F(1)}=\{\varnothing,\{c, d\}\}$, $\mathcal{T}_{F(2)}=\{\varnothing,\{a, c\},\{a\}\}$ and $\mathcal{T}_{F(3)}=\{\varnothing,\{b, d\},\{b\}\}$ and $A=\{1,2,3\} \subset E$. We take the identity function id : $(U, \mathcal{T}) \rightarrow(U, \mathcal{T})$. But, for $1,2 \in A, \mathbf{i d}_{1,2}:\left(F(1), \mathcal{T}_{F(1)}\right) \rightarrow\left(F(2), \mathcal{T}_{F(2)}\right)$ is not continuous function, because $\mathbf{i d}_{1,2}^{-1}[\{a, c\}]=\{a, c\} \notin \mathcal{T}_{F(1)}$ for $\{a, c\} \in \mathcal{T}_{F(2)}$. Hence, id : $(F, A, \mathcal{T}) \rightarrow(F, A, \mathcal{T})$ is not a soft continuous function.

Theorem 3.5. The identity soft function id $:(F, A, \mathcal{T}) \rightarrow(F, A, \mathcal{T})$ is a soft pseudo-continuous function.

Proof. For given the identity soft function id $:(F, A, \mathcal{T}) \rightarrow(F, A, \mathcal{T})$ and for each $p \in A$, id $\mathbf{i d}_{p, p}:\left(F(p), \mathcal{T}_{F(p)}\right) \rightarrow$ $\left(F(p), \mathcal{T}_{F(p)}\right)$ is a continuous function. Thus id is soft pseudo-continuous from Definition 3.1 (1).

We can give the concepts of soft injective and soft surjective function of a given function between two soft sets as follows.

Definition 3.3. Let $\varphi: U \rightarrow V$ be a function and $(F, A)$ and $(G, B)$ are soft sets over $U$ and $V$ respectively. So, 
(1) The function $\varphi:(F, A) \rightarrow(G, B)$ is called a soft injective function if for all $p \in A$ and $p^{\prime} \in B, \varphi_{p, p^{\prime}}: F(p) \rightarrow G\left(p^{\prime}\right)$ is injective.

(2) The function $\varphi:(F, A) \rightarrow(G, B)$ is called a soft surjective function if for all $p \in A$ and $p^{\prime} \in B, \varphi_{p, p^{\prime}}: F(p) \rightarrow$ $G\left(p^{\prime}\right)$ is surjective.

(3) The function $\varphi:(F, A) \rightarrow(G, B)$ is called a soft bijective function if $\varphi$ both soft injective and soft surjective function. follows.

Then, we can give the concepts of soft open and soft closed function and the concept of soft homeomorphism as

Definition 3.4. Let $\varphi:(F, A, \mathcal{T}) \rightarrow\left(G, B, \mathcal{T}^{\prime}\right)$ be a function. Then,

(1) $\varphi$ is called a soft open function if $\varphi_{p, p^{\prime}}:\left(F(p), \mathcal{T}_{F(p)}\right) \rightarrow\left(G\left(p^{\prime}\right), \mathcal{T}_{G\left(p^{\prime}\right)}^{\prime}\right)$ is an open function for each $p \in A$ and $p^{\prime} \in B$.

(2) $\varphi$ is called a soft closed function if $\varphi_{p, p^{\prime}}:\left(F(p), \mathcal{T}_{F(p)}\right) \rightarrow\left(G\left(p^{\prime}\right), \mathcal{T}_{G\left(p^{\prime}\right)}^{\prime}\right)$ is a closed function for each $p \in A$ and $p^{\prime} \in B$.

(3) $\varphi$ is called a soft homeomorphism if it is a soft bijective, soft continuous and soft open or soft closed function. So, we call that $(F, A, \mathcal{T})$ is soft homeomorph to $\left(G, B, \mathcal{T}^{\prime}\right)$ if there is a soft homeomorphism between them.

All properties carried under soft homemorphism are called soft topological properties.

Let $(F, A, \mathcal{T})$ be a soft topological space over $(U, \mathcal{T})$. We know that $(F, A, \mathcal{T})$ is a Hausdorff soft topological space if $\left(F(p), \mathcal{T}_{F(p)}\right)$ is Hausdorff for each $p \in A$ from [9]. We can give the following theorem for the relation between soft continuity and soft Hausdorffness.

Theorem 3.6. Let $\varphi:(F, A, \mathcal{T}) \rightarrow\left(G, B, \mathcal{T}^{\prime}\right)$ be a soft injective and soft continuous function. If $\left(G, B, \mathcal{T}^{\prime}\right)$ is a Hausdorff soft topological space then $(F, A, \mathcal{T})$ is also Hausdorff.

Proof. Since $\varphi_{p, p^{\prime}}:\left(F(p), \mathcal{T}_{F(p)}\right) \rightarrow\left(G\left(p^{\prime}\right), \mathcal{T}_{G\left(p^{\prime}\right)}^{\prime}\right)$ is injective and continuous for each $p \in A$ and $p^{\prime} \in B$, and $\left(G\left(p^{\prime}\right), \mathcal{T}_{G\left(p^{\prime}\right)}^{\prime}\right)$ is Hausdorff, then we obtain that $\left(F(p), \mathcal{T}_{F(p)}\right)$ is Hausdorff for each $p \in A$ from classical theory of topology. This also provides the desired result.

We can clearly obtain the following theorem in parallel with classical theory.

Theorem 3.7. Soft Hausdorffness is a soft topological property.

Proof. The proof is straightforward.

In [9], we define a compact soft topological space as a soft set which the subspaces obtained according to each parameter is a compact subspace. So we can give following theorem for soft continuous functions between compact soft topological spaces.

Theorem 3.8. Let $\varphi:(F, A, \mathcal{T}) \rightarrow\left(G, B, \mathcal{T}^{\prime}\right)$ be a soft continuous and soft surjective function. If $(F, A, \mathcal{T})$ is a compact soft topological space, then $\left(G, B, \mathcal{T}^{\prime}\right)$ is also soft compact.

Proof. Since $\varphi$ is a soft continuous and soft surjective function, then $\varphi_{p, p^{\prime}}:\left(F(p), \mathcal{T}_{F(p)}\right) \rightarrow\left(G\left(p^{\prime}\right), \mathcal{T}_{G\left(p^{\prime}\right)}^{\prime}\right)$ is continuous and surjective for each $p \in A$ and $p^{\prime} \in B$. In addition to this, since $(F, A, \mathcal{T})$ is a soft compact space then the subspaces $\left(F(p), \mathcal{T}_{F(p)}\right)$ are compact for each $p \in A$. Since the image of a compact space under a continuous and surjective function is compact, we obtain that $\left(G\left(p^{\prime}\right), \mathcal{T}_{G\left(p^{\prime}\right)}^{\prime}\right)$ is a compact subspace of $V$ for each $p^{\prime} \in B$. Hence $\left(G, B, \mathcal{T}^{\prime}\right)$ is a compact soft topological space.

In [9], we also define the concept of soft connected soft topological space. Let $(F, A, \mathcal{T})$ be a soft topological space. We call that $(F, A, \mathcal{T})$ is soft connected topological space if $\left(F(p), \mathcal{T}_{F(p)}\right)$ is connected for each $p \in A$. Then we can give following theorem.

Theorem 3.9. Let $\varphi:(F, A, \mathcal{T}) \rightarrow\left(G, B, \mathcal{T}^{\prime}\right)$ be a soft continuous and soft surjective function. If $(F, A, \mathcal{T})$ is a connected soft topological space, then $\left(G, B, \mathcal{T}^{\prime}\right)$ is also connected.

Proof. The proof is straightforward. 


\section{An Application Method: Ordering The Parameters and a Decision-Making Technique in Soft Topological Space}

We know that a soft set is one of the very important and very useful mathematical tool in decision-making problems. Many scientists have studied and developed several techniques in this field. We recommend that you review the articles in $[5,6,8,10,12,15]$ to see these methods and techniques. The soft set theory can easily model a decision-making process. We need to choose the right parameters to ensure that we make the right decision because all the decisions we make depend on the parameters. But the excess of the parameters adversely affects the decision-making process. We have to choose the most appropriate and important parameters for the decisionmaking process before deciding. In this section, we will classify the specific parameters using soft topological concepts and we will provide a decision-making technique accordingly.

The concepts of interior and closure of a set are useful and important topological concepts in a topological space. We use these concepts classifying the parameters in the soft set $(F, A)$. We have already mentioned the overt soft topological space over a topological space. Namely, let $(U, \mathcal{T})$ be a topological space and $\widetilde{\mathbb{U}}_{A}$ be a whole soft set over $U$, then we obviously have a soft topological space $\left(\widetilde{\mathbb{U}}_{A}, \mathcal{T}\right)$ which is called overt soft topological space. Suppose that $(F, B) \widetilde{\subset} \widetilde{\mathbb{U}}_{A}$. Now, let's define some specific parameters using topological concepts.

Definition 4.1. (a) The parameter $p \in B$ is called core parameter if $\mathfrak{i}(F(p)) \neq \varnothing$.

(b) The parameter $p \in B$ is called indispensable parameter if $\mathfrak{c}(F(p))=U$.

(c) The parameter $p \in B$ is called dispensable parameter if $\mathfrak{i}(F(p))=\varnothing$

Example 4.1. Let $U=\{a, b, c, d, e\}$ be a set and $\mathcal{T}=\{\varnothing, U,\{a, b\},\{a, c\},\{a\},\{a, b, c\}\}$ be a topology on $U$. The overt soft topological space is $\left(\widetilde{\mathbb{U}}_{A}, \mathcal{T}\right)$ where $A=\{1,2,3,4,5,6\}$. Let $(F, B)=\{1=\{a, e\}, 4=\{a, b, d\}, 6=\{b, e\}\}$ be a soft subset of $\widetilde{\mathbb{U}}_{A}$. Since $\mathfrak{i}(F(1))=\{a\} \in \mathcal{T}, 1 \in B$ is a core parameter. Since $\mathfrak{c}(F(4))=U, 4 \in B$ is indispensable parameter. Since $\mathfrak{i}(F(6))=\varnothing, 6 \in B$ is a dispensable parameter.

Using the Definition 4.1, we can make a comparison between the parameters. If we have core, indispensable and dispensable parameters, we can say that the core parameter is superior than the dispensable parameter, and the indispensable parameter is superior than the core parameter. According to comparison we give here, we have the following schema in Example 4.1.

$$
6 \prec 1 \prec 4 .
$$

Theorem 4.1. Let $(F, B)$ be a soft subset of the overt soft topological space $\left(\widetilde{\mathbb{U}}_{A}, \mathcal{T}\right)$.

1. If $(F, B)$ is a non-empty approximated quasi-open soft set then there exist a core parameter.

2. If $(F, B)$ is a non-empty approximated open soft set then all parameters in $B$ are core parameters.

3. If $(F, B)$ is a everywhere dense soft set then all parameters in $B$ are indispensable parameters.

The proof of Theorem 4.1 is obvious.

We can get rid of the parameter stack in the decision-making system by using the definitions of these special parameters, that is, we can make a kind of parameter reduction. The parameters that are important for decision in the system are the core and indispensable parameters. Since dispensable parameters are useless, we can discard these parameters from the system. So we get a system with fewer parameters. Now, using this reduced system, it will be easier to make a decision.

If we have a topological space in which the system we will decide, then, as a decision-making method, we can follow these steps.

Let $(U, \mathcal{T})$ be a topological space, and $\left(\widetilde{\mathbb{U}}_{A}, \mathcal{T}\right)$ be the overt soft topological space (of course, an arbitrary soft topological space can also be taken). Suppose that $(F, B)$ is a soft subset of $\widetilde{\mathbb{U}}_{A}$. If the conditions mentioned above are provided, we can make a decision on the system in two steps

Step 1 If there are core and indispensable parameters, they are selected and parameter reduction is done by removing the remaining unnecessary parameters.

Step 2 The intersection of approximated sets is taken over all parameters in the reduced soft set, i.e. suppose that $\left(F, B^{*}\right)$ is reduced form of $(F, B)$, then we select an element in $\bigcap_{p \in B^{*}} F(p)$. 
As a result, the smallest approximate set satisfying all the conditions of core and indispensable parameters is obtained. The decision-maker selects an element from this set.

Example 4.2. Consider the argument of Example 4.1. So we obtain a reduced system as follows,

$$
\left(F, B^{*}\right)=\{1=\{a\}, 4=\{a, b, d\}\} .
$$

Thus, in this abstract universe, the decision-maker with this fiction will choose the element $a$.

So we have developed a method by which topology can be directly applied to everyday life problems.

\section{Conclusion}

In this article, we have given some other topological concepts for soft topological spaces using the spirit given in [9]. Surely, this is a spirit that preserves Molodtsov's soft set sense. We have defined the concepts of open soft set, closed soft set, interior and closure of a soft set, derived soft set, perfect soft set and dense soft set in a soft topological space and discussed some of its structural properties. Moreover, we have defined the concept of continuity and homeomorphism between two soft topological spaces. Using the concepts of Hausdorffness, compactness and connectedness, given in [9], we have given some properties under soft continuous functions. Finally, we propose a decision-making method as an application method using soft topological concepts. In future work, using the basic concepts given in [9] and here, the topological concepts such as sequence and filter can be studied in soft topological spaces.

The author hope that this article sheds light on a way of working scientists in this field.

\section{Acknowledgment}

The author thanks the anonymous referees and editors for their constructive comments, criticisms and advices.

\section{References}

[1] Acar, U., Koyuncu, F., Tanay, B.: Soft sets and soft rings. Comput. Math. Appl. 59 (11), 3458-3463 (2010).

[2] Aktaş, H., Çağman, N.: Soft sets and soft groups. Inform. Sci. 177 (13), 2726-2735 (2007).

[3] Ali, M.I., Feng, F., Liu, X., Min, W.K., Shabir, M.: On some new operations in soft set theory. Comput. Math. Appl. 57, 1547-1553 (2009).

[4] Aygüoğlu, A., Aygün, H.: Some notes on soft topological spaces. Neural Comput. \& Applic. 21 (1), 113-119 (2012).

[5] Çağman, N., Enginoğlu, S.: Soft set theory and uni - int decision making. European J. Oper. Res. 207 (2), 848-855 (2010).

[6] Çetkin, V., Aygüoğlu, A., Aygün, H.: A new approach in handling soft decision making problems. J. Nonlinear Sci. Appl. 9, 231-239 (2016).

[7] Hussain, S., Ahmad, B.: Some properties of soft topological spaces. Comput. Math. Appl. 62 (11), 4058-4067 (2011).

[8] Kandemir, M.B.: Monotonic soft sets and its applications. Ann. Fuzzy Math. Inform. 12 (2), 295-307 (2016).

[9] Kandemir, M.B.: A new perspective on soft topology. Hittite J. Sci. Eng. 5 (2), 105-113 (2018).

[10] Kandemir, M.B.: The concept of $\sigma$-algebraic soft set. Soft Comput. 22 (13), 4353-4360 (2018).

[11] Maji, P.K., Biswas, R., Roy, A.R.: Soft set theory. Comput. Math. Appl. 45, 555-562 (2003).

[12] Maji, P.K., Roy, A.R., Biswas, R.: An application of soft sets in a decision making problem. Comput. Math. Appl. 44 (8-9), 1077-1083 (2002).

[13] Molodtsov, D.: Soft set theory - first results. Comput. Math. Appl. 37, 19-31 (1999).

[14] Munkres, J.R.: Topology. 2nd Ed. Prentice Hall, Inc., (2000). 
[15] Qin, K., Yang, J., Zhang, X.: Soft set approaches to decision making problems. In. T. Li et al. (eds) Rough Sets and Knowledge Technology RSTK 2012. Lecture Notes in Computer Science, vol. 7414, Springer, 456-464 (2012).

[16] Shabir, M., Naz, M.: On soft topological spaces. Comput. Math. Appl. 61 (7), 1786-1799 (2011).

[17] Yüksel, Ş.: General topology. 5th, extended edition. Eğitim Kitabevi Yayınları, (2006) (In Turkish).

\section{Affiliations}

Mustafa BuRÇ KANDEMIR

AddREsS: Mugla Sitkı Koçman University, Department of Mathematics, 48000, Mugla-Turkey.

E-MAIL: mbkandemir@mu.edu.tr

ORCID ID: 0000-0002-0159-5670 